\title{
Duality Relations for Potts Correlation Functions
}

\author{
F. Y. Wu \\ Department of Physics, Northeastern University, Boston, Massachusetts 02115, U. S. A. \\ Centre Emile Borel - UMS 839 IHP (CNRS/UPMC) - Paris, France
}

\begin{abstract}
Duality relations are obtained for correlation functions of the $q$-state Potts model on any planar lattice or graph using a simple graphical analysis. For the two-point correlation we show that the correlation length is precisely the surface tension of the dual model, generalizing a result known to hold for the Ising model. For the three-point correlation an explicit expression is obtained relating the correlation function to ratios of dual partition functions under fixed boundary conditions.
\end{abstract}




\section{INTRODUCTION}

It is now well-known [1,2] that the correlation length of the Ising model in two dimensions is precisely the surface tension of the dual lattice. It is also known by folklore (see, for example, [3]) that a similar duality exists for the Potts model [4]. However, detailed discussion of the correlation duality for the Potts model has yet to appear in the literature. In view of the important role played by such duality relations in discussions of the equilibrium crystal shapes [5],6], a definitive understanding of this subject matter is clearly needed.

Here we take up this question and consider more generally the duality for the $n$-point correlation function. On the basis of a simple graphical analysis, we derive duality relations for the two- and three-point correlation functions. Particularly, we establish that the correlation length in the large lattice limit is precisely the surface tension of the dual model, thus generalizing the known Ising result, and that the three-point correlation function is given

by similarly defined expressions in the dual space. Our analysis, which does not concern with the internal structure of the underlying lattice, is quite general and applies to the Potts model with arbitrary edge-dependent interactions and/or on any planar lattice or graph. It can also be extended to higher correlations.

Consider a $q$-state Potts model [4] on a two-dimensional lattice $L$ with a free boundary. An example of $L$ is the rectangular $4 \times 6$ lattice shown in Fig. 1(a), but more generally $L$ can be any planar lattice or graph which does not need to be regular. The site $i$ of $L$ is occupied by a spin $\sigma_{i}$ which can take on values $1,2, \ldots, q$. Two spins at sites $\alpha$ and $\beta$ and in states $\sigma_{\alpha}$ and $\sigma_{\beta}$ interact with a Boltzmann factor $\exp \left[K_{\alpha \beta} \delta\left(\sigma_{\alpha}, \sigma_{\beta}\right)\right]$. Let $i, j, \cdots, n$ denote any $n$ sites on the boundary. We introduce the $n$-point correlation function as the probability that the spins at sites $\{i, j, \cdots, n\}$ are in the respective states $\left\{\sigma, \sigma^{\prime}, \cdots, \sigma^{(n-1)}\right\}$,

$$
P_{n}\left(\sigma, \sigma^{\prime}, \cdots, \sigma^{(n-1)}\right)=<\delta\left(\sigma_{i}, \sigma\right) \delta\left(\sigma_{j}, \sigma^{\prime}\right) \cdots \delta\left(\sigma_{n}, \sigma^{(n-1)}\right)>
$$

where $<\cdot>$ denotes statistical averages. It is sometimes convenient to consider the correlation function (see, for example [7, 3] for $n=2$ ) 


$$
\begin{aligned}
\Gamma_{n}\left(\sigma_{i}, \sigma_{j}, \cdots, \sigma_{n}\right) & =<q^{n-1} \delta\left(\sigma_{i}, \sigma_{j}\right) \delta\left(\sigma_{j}, \sigma_{k}\right) \cdots \delta\left(\sigma_{n-1}, \sigma_{n}\right)-1> \\
& =q^{n} P_{n}(\sigma, \sigma, \cdots, \sigma)-1,
\end{aligned}
$$

a quantity which vanishes identically if the $n$ spins $\left\{\sigma_{i}, \sigma_{j}, \sigma_{k} \cdots, \sigma_{n-1}, \sigma_{n}\right\}$ are completely uncorrelated. In the case of $q=n=2$, for example, one writes $2 \delta\left(\sigma_{i}, \sigma_{j}\right)=1+\sigma_{i} \sigma_{j}$ where $\sigma_{i, j}= \pm 1$, then (2) becomes the usual expression $\Gamma_{2}\left(\sigma_{i}, \sigma_{j}\right)=<\sigma_{i} \sigma_{j}>$ for the Ising model. It is clear that the correlation function $P_{n}$ is more general than $\Gamma_{n}$.

Our analyses is based on the repeated use of a fundamental duality relation for the Potts model [7]. Construct $L^{*}$, the dual lattice (graph) of $L$, by placing spins in the faces of $L$ including the exterior one. In Fig. 1(a), for example, the faces of the dual lattice are denoted by crosses and the letter $s$, so $L^{*}$ has $N^{*}=15+1=16$ sites. Edges of $L^{*}$ (not shown in Fig. $1(\mathrm{a}))$ bisect, and are in one-one correspondence with, edges of $L$. Let $K_{\alpha \beta}^{*}$ be the interaction of the edge bisecting the interaction $K_{\alpha \beta}$. Let $Z\left(\left\{K_{\alpha \beta}\right\}\right)$ and $Z^{*}\left(\left\{K_{\alpha \beta}^{*}\right\}\right)$ be the respective partition functions on $L$ and $L^{*}$. Then one has the following fundamental duality relation |7]

$$
Z\left(\left\{K_{\alpha \beta}\right\}\right)=q C Z^{*}\left(\left\{K_{\alpha \beta}^{*}\right\}\right)
$$

where $K_{\alpha \beta}$ and $K_{\alpha \beta}^{*}$ are related by

$$
\left(e^{K_{\alpha \beta}}-1\right)\left(e^{K_{\alpha \beta}^{*}}-1\right)=q,
$$

and $C=q^{-N^{*}} \prod_{\text {edges }}\left(e^{K_{\alpha \beta}}-1\right)$.

\section{THE TWO-POINT CORRELATION FUNCTION}

Let $i$ and $j$ denote any two sites on the boundary of $L$ as shown in Fig. 1(a). Further let

$$
Z_{11}=Z\left(\sigma_{i}=\sigma_{j}=1\right), \quad Z_{12}=Z\left(\sigma_{i}=1, \sigma_{j}=2\right)
$$

be the partition functions with $\sigma_{i}$ and $\sigma_{j}$ fixed in definite states 1 and/or 2, Then, by symmetry we have 


$$
Z\left(\left\{K_{\alpha \beta}\right\}\right)=q Z_{11}+q(q-1) Z_{12} .
$$

Clearly, we also have $P_{2}(1,1)=Z_{11} / Z$ and $P_{2}(1,2)=Z_{12} / Z$.

Let $Z_{11}^{*}$ denote the dual partition function with the spin in the exterior face in a definite state, say, $s$. Alternately, from Fig. 1(a), we see that $Z_{11}^{*}$ can also be regarded as the dual partition function with all boundary spins interact with the exterior spin fixed at state $s$. Then we have

$$
Z^{*}\left(\left\{K_{\alpha \beta}\right\}\right)=q Z_{11}^{*}
$$

Substituting (6) and (7) into the duality relation (3), one obtains the relation

$$
Z_{11}+(q-1) Z_{12}=q C Z_{11}^{*}
$$

To obtain a second relation relating $Z_{11}$ and $Z_{12}$, we apply the duality relation (3) to a lattice, or graph, constructed from $L$ by adding one additional edge connecting sites $i$ and $j$ with an interaction $K$, a situation shown in Fig. 1(c). Clearly, the respective partition functions for the new lattice and its dual are now

$$
\tilde{Z}=q e^{K} Z_{11}+q(q-1) Z_{12}
$$

and

$$
\tilde{Z}^{*}=q e^{K^{*}} Z_{11}^{*}+q(q-1) Z_{12}^{*}
$$

where $Z_{12}^{*}$ is the partition function of the dual under the boundary condition such that the spins in the two exterior faces are in definite states $s \neq s^{\prime}$ as shown in Fig. 1(b). Here, $K$ and $K^{*}$ are related by (4). Substituting (9) and (10) into (3) and noting that $N^{*}$ is increased by 1 in the new lattice, one obtains a second relation

$$
e^{K} Z_{11}+(q-1) Z_{12}=C\left(e^{K}-1\right)\left[e^{K^{*}} Z_{11}^{*}+(q-1) Z_{12}^{*}\right] .
$$

We now solve (8) and (11) for $Z_{11}$ and $Z_{12}$. This leads to, after using (国) to eliminate $e^{K^{*}}$, 


$$
\begin{aligned}
& Z_{11}=C\left[Z_{11}^{*}+(q-1) Z_{12}^{*}\right] \\
& Z_{12}=C\left(Z_{11}^{*}-Z_{12}^{*}\right) .
\end{aligned}
$$

Note that the interaction $K$ introduced to facilitate calculations does not enter (12). The expression (12) now leads to the desired expression for the correlation function. Particularly, using the identity $Z=q^{2} C Z_{11}^{*}$, we obtain

$$
P_{2}\left(\sigma, \sigma^{\prime}\right)=\frac{1}{q^{2}}\left[1+\left(q \delta_{\sigma, \sigma^{\prime}}-1\right) \frac{Z_{12}^{*}}{Z_{11}^{*}}\right]
$$

and

$$
\Gamma_{2}\left(\sigma_{i}, \sigma_{j}\right)=(q-1)\left(\frac{Z_{12}^{*}}{Z_{11}^{*}}\right)
$$

For the Potts model the surface tension $\tau$ is defined by [9]

$$
\tau=-\lim _{D \rightarrow \infty} \frac{1}{D} \ln \left(\frac{Z_{12}^{*}}{Z_{11}^{*}}\right)
$$

where $D$ is the distance between sites $i$ and $j$, the two points where the boundary spin state changes from $s$ to $s^{\prime}$ in Fig. 1(b). Our result (14) now relates the correlation function on $L$ to the surface tension on $L^{*}$. Particularly, the known exponential decay $e^{-D / \xi}$ of the two-point correlation $\Gamma_{2}$ above the transition temperature $T_{c}$ [10], where $\xi$ is the correlation length, leads to the identity $\xi^{-1}=\tau$, where $\tau$ is the surface tension of the dual model below $T_{c}$. This generalizes the corresponding $q=2$ result of the Ising model [1, 2, to all $q$.

\section{THE THREE-POINT CORRELATION}

Consider any three sites $i, j, k$ on the boundary of $L$ as shown in Fig. 2. Here, to emphasize the generality of our consideration and the fact that the internal structure of $L$ does not enter the picture, the lattice is shown symbolically as a shaded region, Let

$$
Z_{\sigma \sigma^{\prime} \sigma^{\prime \prime}}=Z\left(\sigma_{i}=\sigma, \sigma_{j}=\sigma^{\prime}, \sigma_{j}=\sigma^{\prime \prime}\right) . \quad \sigma, \sigma^{\prime}, \sigma^{\prime \prime}=1,2, \ldots, q
$$

denote the partition function with $\sigma_{i}, \sigma_{j}, \sigma_{k}$ fixed in definite states. Then, in analogous to (6) the partition function can be written as 


$$
Z=q Z_{111}+q(q-1)\left[Z_{211}+Z_{121}+Z_{112}\right]+q(q-1)(q-2) Z_{123}
$$

In a similar manner, let $Z_{s s^{\prime} s^{\prime \prime}}^{*}$ be the partition function of the dual model under a boundary condition such that all boundary spins between sites $i$ and $j$ of $L$ interact with a fixed spin state $s^{\prime \prime}$, all boundary spins between $j$ and $k$ interact with a spin state $s$, and all boundary spins between $k$ and $i$ interact with a spin state $s^{\prime}$, a situation shown in Fig. 2. Then, the dual partition function can be written as

$$
Z^{*}=q Z_{111}^{*}
$$

Substituting (17) and (18) into (3), one obtains the relation

$$
Z_{111}+(q-1)\left(Z_{211}+Z_{121}+Z_{112}\right)+(q-1)(q-2) Z_{123}=q C Z_{111}^{*}
$$

Next we modify $L$ by connecting sites $j$ and $k$ with a new edge and apply the result (12). In the first line of (12), one has, by definition,

$$
\begin{aligned}
& Z_{11}=\sum_{i=1}^{q} Z_{i 11}=Z_{111}+(q-1) Z_{211} \\
& Z_{11}^{*}=Z_{111}^{*} \\
& Z_{12}^{*}=Z_{122}^{*}=Z_{211}^{*} .
\end{aligned}
$$

Substituting (20) into (12), one obtains a second relation

$$
Z_{111}+(q-1) Z_{211}=C\left[Z_{111}^{*}+(q-1) Z_{211}^{*}\right]
$$

In a similar manner by connecting sites $\{k, i\}$ or $\{i, j\}$ with a new edge, we obtain, respectively,

$$
\begin{aligned}
& Z_{111}+(q-1) Z_{121}=C\left[Z_{111}^{*}+(q-1) Z_{121}^{*}\right] \\
& Z_{111}+(q-1) Z_{112}=C\left[Z_{111}^{*}+(q-1) Z_{112}^{*}\right]
\end{aligned}
$$

Finally, we apply the duality relation (3) to a lattice constructed by introducing 3 new edges connecting sites $i, j$ or $k$ to a common new point with interactions $K$ as shown in Fig. 2 . This process increases $N^{*}$ by 2 and yields the duality relation 


$$
\begin{gathered}
\left(e^{3 K}+q-1\right) Z_{111}+(q-1)\left(e^{2 K}+e^{K}+q-2\right)\left(Z_{211}+Z_{121}+Z_{112}\right) \\
+(q-1)(q-2)\left(3 e^{K}+q-3\right) Z_{123} \\
=C\left(e^{K}-1\right)^{3} q^{-1}\left[e^{3 K^{*}} Z_{111}^{*}+(q-1) e^{K^{*}}\left(Z_{211}^{*}+Z_{121}^{*}+Z_{112}^{*}\right)\right. \\
\left.+(q-1)(q-2) Z_{123}^{*}\right] .
\end{gathered}
$$

From (19), (21), (22), (23), and (24) and using (4) to eliminate $K^{*}$, we solve $Z_{111}, Z_{211}$, $Z_{121}, Z_{112}$, and $Z_{123}$ in terms of the dual partition functions $Z_{s s^{\prime} s^{\prime \prime}}^{*}$ This leads to

$$
\begin{aligned}
& Z_{111}=(C / q)\left[Z_{111}^{*}+(q-1)(q-2) Z_{123}^{*}+(q-1)\left(Z_{211}^{*}+Z_{121}^{*}+Z_{112}^{*}\right)\right] \\
& Z_{123}=(C / q)\left[Z_{111}^{*}+2 Z_{123}^{*}-Z_{211}^{*}-Z_{121}^{*}-Z_{112}^{*}\right] \\
& Z_{211}=(C / q)\left[Z_{111}^{*}-(q-2) Z_{123}^{*}+(q-1) Z_{211}^{*}-Z_{121}^{*}-Z_{112}^{*}\right] \\
& Z_{121}=(C / q)\left[Z_{111}^{*}-(q-2) Z_{123}^{*}-Z_{211}^{*}+(q-1) Z_{121}^{*}-Z_{112}^{*}\right] \\
& Z_{112}=(C / q)\left[Z_{111}^{*}-(q-2) Z_{123}^{*}-Z_{211}^{*}-Z_{121}^{*}+(q-1) Z_{112}^{*}\right] .
\end{aligned}
$$

This result is again independent of the parameter $K$ used in the evaluation.

Using (25) and the identity $Z=C q Z^{*}=C q^{2} Z_{111}^{*}$, we finally obtain

$$
\begin{gathered}
P_{3}\left(\sigma, \sigma^{\prime}, \sigma^{\prime \prime}\right)=\frac{1}{q^{3}}\left[1+2 p_{123}-\left(p_{211}+p_{121}+p_{112}\right)+q\left(p_{211}-p_{123}\right) \delta_{\sigma^{\prime}, \sigma^{\prime \prime}}\right. \\
\left.+q\left(p_{121}-p_{123}\right) \delta_{\sigma^{\prime \prime}, \sigma}+q\left(p_{112}-p_{123}\right) \delta_{\sigma, \sigma^{\prime}}+q^{2} p_{123} \delta_{\sigma, \sigma^{\prime}} \delta_{\sigma^{\prime}, \sigma^{\prime \prime}}\right]
\end{gathered}
$$

and

$$
\begin{aligned}
\Gamma_{3}\left(\sigma_{i}, \sigma_{j}, \sigma_{k}\right) & =(q-1)\left[p_{112}+p_{121}+p_{211}+(q-2) p_{123}\right] \\
& =\Gamma_{2}\left(\sigma_{i}, \sigma_{j}\right)+\Gamma_{2}\left(\sigma_{j}, \sigma_{k}\right)+\Gamma_{2}\left(\sigma_{k}, \sigma_{i}\right)+(q-1)(q-2) p_{123} .
\end{aligned}
$$

Here,

$$
p_{s s^{\prime} s^{\prime \prime}}=Z_{s s^{\prime} s^{\prime \prime}}^{*} / Z_{111}^{*}
$$

is the ratio of dual partition functions which can be interpreted as appropriately defined surface tensions, and we have used $(q-1) p_{112}=\Gamma_{2}\left(\sigma_{i}, \sigma_{j}\right)$, etc. Since $Z^{*}=q Z_{111}^{*}$, the ratio 
$p_{s s^{\prime} s^{\prime \prime}}$ gives also the probability that the particular boundary condition $\left\{s, s^{\prime}, s^{\prime \prime}\right\}$ of Fig. 2 occurs in the dual partition function $Z^{*}$. It is readily verified that the identity

$$
\sum_{\sigma^{\prime \prime}} P_{3}\left(\sigma, \sigma^{\prime}, \sigma^{\prime \prime}\right)=P_{2}\left(\sigma, \sigma^{\prime}\right)
$$

is satisfied.

\section{SUMMARY}

In summary, we have presented a graphical analysis of duality relations leading to explicit expressions for correlation functions of the $q$-state Potts model on any planar lattice or graph. For the two-point correlation our result (14) generalizes a relation previously known for the Ising model. For three-point correlations, our result (26) is new and can be used to compute any three-point correlation. In all cases the correlation functions are found to be given by ratios of dual partition functions under fixed boundary conditions, which can in turn be interpreted as appropriately defined surface tensions. Our consideration can be extended in a straightforward fashion to higher correlations $P_{n}$ and $\Gamma_{n}$ for $n>3$, and to the more general $\left(N_{\alpha}, N_{\beta}\right)$ model [11] including the $N_{\alpha}=N_{\beta}=2$ Ashkin-Teller model [12].

\section{ACKNOWLEDGEMENTS}

I would like to thank R. K. P. Zia for suggesting this problem and enlightening discussions. I would also like to thank M.-J. Maillard for the hospitality at the Institut Henri Poincaré and H. Kunz for the hospitality at Institut de Physique Théorique, Lausanne,

where this research was initiated. This work is supported in part by the National Science Foundation Grant DMR-9614170. 


\section{REFERENCES}

[1] P. G. Watson, J. Phys. C1 (1968) 575.

[2] R. K. P. Zia, Phys. Lett. 64A (1978) 345.

[3] F. Y. Wu, Rev. Mod. Phys. 54 (1982) 235.

[4] R. B. Potts, Proc. Camb. Phil. Soc. 48, (1952) 106.

[5] C. Rottman and M. Wortis, Phys. Rev. B24 (1981) 6274.

[6] J. E. Avron, H. van Beijeren, and R. K. P. Zia, J. Phys. A15 (1982) L81.

[7] F. Y. Wu and Y. K. Wang, J. Math. Phys. 17 (1976) 439.

[8] Y. K. Wang and F. Y. Wu, J. Phys. A9 (1976) 593.

[9] J. R. Fontaine and Ch. Gruber, Commun. Math. Phys. 70 (1979) 243.

[10] A. H. Hintermann, H. Kunz, and F. Y. Wu, J. Stat. Phys. 19 (1978) 623.

[11] E. Domany and E. K. Riedel, Phys. Rev. Lett. 40 (1978) 561.

[12] J. Ashkin and E. Teller, Phys. Rev. 64 (1943) 178. 


\section{FIGURES}

FIG. 1. (a) A $4 \times 6$ lattice $L$. The dual spins are denoted by crosses and the letter $s$. (b) The boundary condition $\left(s \neq s^{\prime}\right)$ for $Z_{12}^{*}$. (c) The addition of one edge to $L$ connecting sites $i$ and $j$.

FIG. 2. The addition of 3 edges to $L$ (the shaded region) connecting sites $i, j$, and $k$ to a common point. 


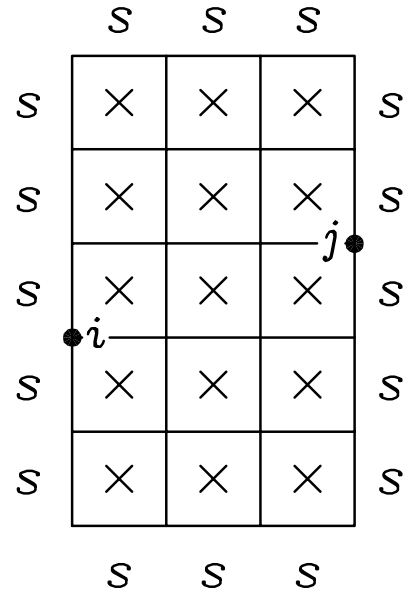

(a)

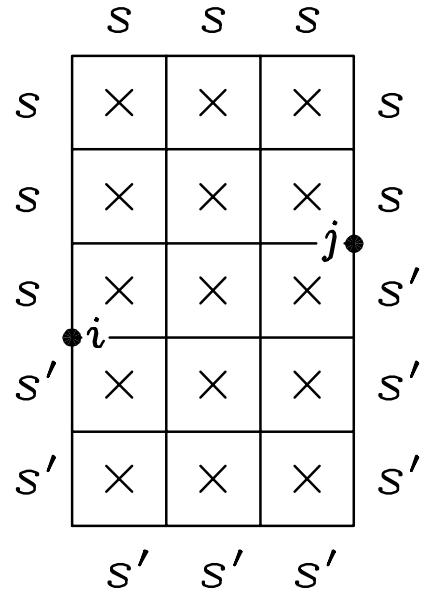

(b)

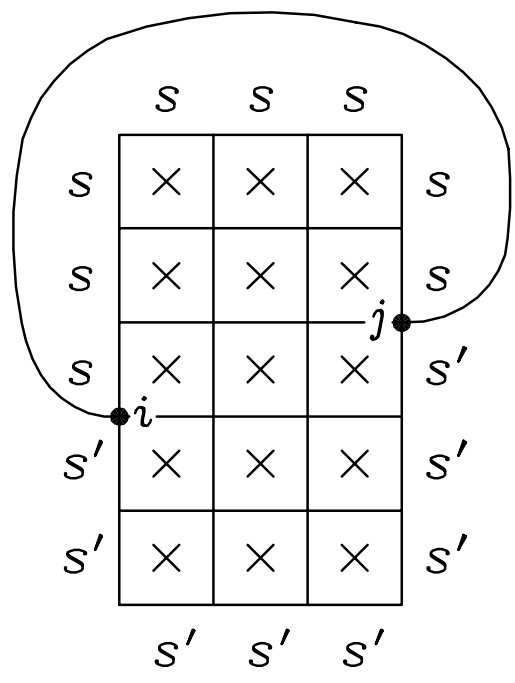

(c)

Fig. 1 


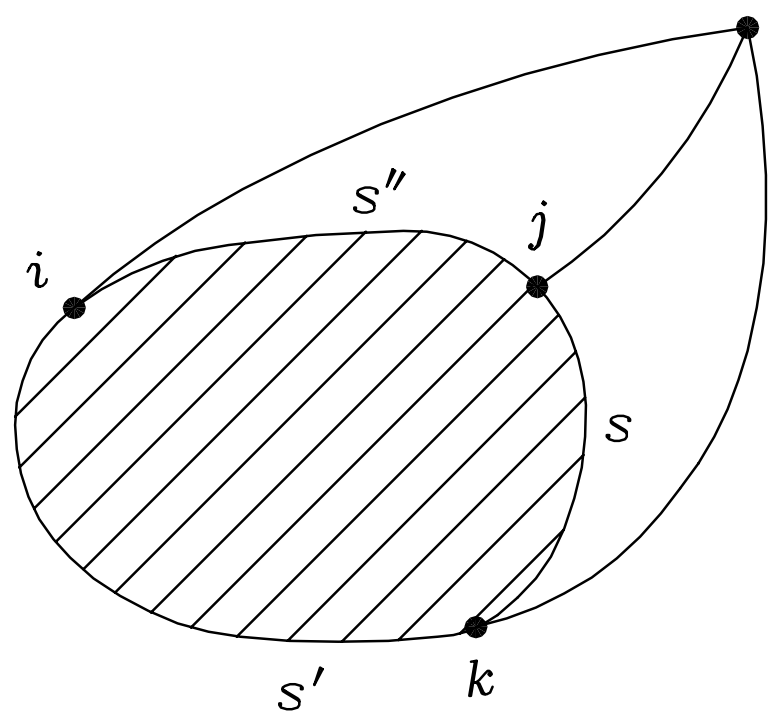

Fig. 2 